\title{
Exosome long non-coding RNA SOX2-OT contributes to ovarian cancer malignant progression by miR-181b-5p/SCD1 signaling
}

\author{
Yongjing Lai ${ }^{1}$, Lihua Dong ${ }^{1}$, Huifang Jin ${ }^{1}$, Hongju Li ${ }^{2}$, Meiling Sun ${ }^{1}$, Jianlan Li ${ }^{3}$ \\ ${ }^{1}$ Department of Obstetrics, People's Hospital of Rizhao, Rizhao, China \\ ${ }^{2}$ Department of Health Emergency Office, Rizhao Center for Disease Control and Prevention, Rizhao, China \\ ${ }^{3}$ Department of Gynaecolgy, People's Hospital of Rizhao, Rizhao, China
}

Correspondence to: Jianlan Li; email: lanlmcpz201@163.com, https://orcid.org/0000-0003-1677-517X

Keywords: ovarian cancer, progression, SOX2-OT, exosome, miR-181b-5p

Received: November 24, $2020 \quad$ Accepted: May 11, $2021 \quad$ Published: October 24, 2021

Copyright: (C) 2021 Lai et al. This is an open access article distributed under the terms of the Creative Commons Attribution License (CC BY 3.0), which permits unrestricted use, distribution, and reproduction in any medium, provided the original author and source are credited.

\section{ABSTRACT}

Ovarian cancer is a common gynecologic cancer with increased mortality and morbidity. Exosome-delivered long non-coding RNAs have been well found in cancer development. However, the function of exosomal SOX2-OT in ovarian cancer development is still unreported. In the present study, we were interested in the investigation of the effect of exOsomal SOX2-OT during ovarian cancer pathogenesis. Significantly, we revealed that the SOX2-OT expression levels were up-regulated in the ovarian cancer patients' plasma exosomes. The depletion of exosomal SOX2-OT inhibited migration, invasion, and proliferation and induced apoptosis in ovarian cancer cells. In mechanical exploration, SOX2-OT could sponge miR-181b-5p, and miR181b-5p was able to target SCD1 in the ovarian cancer cells. The SCD1 overexpression and miR-181b-5p inhibitor could reverse exosomal SOX2-OT-mediated ovarian cancer progression. Functionally, the depletion of exosomal SOX2-OT significantly reduced tumor growth of ovarian cancer cells in vivo. In summary, we concluded that exosomal SOX2-OT enhanced ovarian cancer malignant phenotypes by miR-181b-5p/SCD1 axis. Our finding presents novel insights into the mechanism by which exosomal IncRNA SOX2-OT promotes ovarian cancer progression. SOX2-OT, miR-181b-5p, and SCD1 may serve as potential targets for the treatment of ovarian cancer.

\section{INTRODUCTION}

Ovarian cancer serves as the primary reason for women cancer-related mortality in all gynecologic tumors [1]. Despite therapies have been advanced, treatments are limited because of drug-resistance [2]. The diagnosis normally occurs at the more advanced stage in ovarian cancer patients [3]. Current treatment is not available to cure ovarian cancer, and 5-year survival incidence maintains at $45 \%$ [4]. Accordingly, understanding the molecular mechanism of ovarian cancer progression will be helpful for the improvement of therapeutic strategies for ovarian cancer [5-7]. However, the investigation of the molecular mechanism of ovarian cancer development is still limited.
The 30-100 nm nano-sided particles termed exosomes are extracellularly delivered after multivesicular endosome fusion $[8,9]$. The exosomes transfer long non-coding RNAs, microRNA (miRNAs), (lncRNAs), and some active materials, playing crucial roles in the modulation of cancer pathogenesis [10, 11]. Exosomes-derived non-coding RNAs have been identified to participate in ovarian cancer progression $[12,13]$. Meanwhile, IncRNA SOX2-OT is able to load in the exosome and is involved in the regulation of multiple cancers, including prostate cancer, nasopharyngeal carcinoma, and lung squamous cell carcinoma [14-16]. Meanwhile, it has been identified the clinical significance of IncRNA SOX2-OT in ovarian cancer cells and clinical tissues [17]. SOX2- 
OT contributes to motility and proliferation of ovarian cancer cells (Han, 2018 \#25). However, the function of exosomal SOX2-OT during ovarian cancer development is still unreported. Hence, the exploration of the exosomal SOX2-OT effect on the ovarian cancer progression is innovative.

MicroRNAs (miRNAs) presents fundamental roles in many cellular mechanisms via regulating numerous genes at the post-transcriptional level [18, 19]. Several miRNAs are involved in the tumorigenesis of ovarian cancer. For instance, MiR-126-3p decreases invasion and proliferation by inhibiting PLXNB2 in ovarian cancer [20]. MiR-34a inhibits chemoresistance and cell proliferation of ovarian cancer by regulating HDAC1 [21]. Meanwhile, miR-181b-5p serves as a tumor suppressor for multiple cancers, such as prostate cancer, non-small cell lung cancer, and colorectal cancer [22-24]. Moreover, sterol CoA desaturase (SCD1), a lipid regulating enzyme, is upregulated in cancer cells to facilitate malignancies, containing liver cancer, lung cancer, breast cancer [25-27]. Besides, it has been found that SCD1 is able to regulate ferroptosis in ovarian cancer [28]. Nevertheless, the effect of exosomal SOX2-OT on miR-181b-5p and SCD1 during ovarian cancer pathogenesis remains unclear. Consequently, it is innovative to investigate the function of exosomal SOX2-OT/miR-181b-5p/SCD1 signaling in ovarian cancer development.

In this study, we focused on the exploration of exosomal SOX2-OT function in ovarian cancer. We uncovered the new role of exosomal SOX2-OT in enhancing ovarian cancer malignant phenotype by modulating miR-181b-5p/SCD1 axis.

\section{RESULTS}

\section{The expression of SOX2-OT is enhanced in the plasma exosome of ovarian cancer patients}

Firstly, we performed the lncRNA profiling in the exosomes from ovarian cancer patients $(\mathrm{n}=5)$ and normal samples $(\mathrm{n}=5)$ and identified the elevated expression of SOX2-OT in the exosomes from ovarian cancer patients compared with normal samples (Figure 1A). To assess the potential correlation of exosomal SOX2-OT with the ovarian cancer progression, we analyzed their expression in the plasma exosome of ovarian cancer patients. Significantly, the expression of SOX2-OT was enhanced in the plasma exosome from ovarian cancer patients $(n=55)$ relative to the normal cases $(\mathrm{n}=55)$ (Figure 1B). In addition, ovarian cancer patient tissues $(\mathrm{n}=55)$ presented higher expression of SOX2-OT compared with the adjacent normal tissues $(\mathrm{n}=55)$ (Figure 1C). Furthermore, TEM analysis revealed that exosomes from the ovarian cancer patients showed the same size as the normal cases (Supplementary Figure 1A). Similarly, the existence of the exosome markers, such as TSG101 and CD63, in the exosome of ovarian cancer patients and normal cases, was validated (Supplementary Figure 1B).

\section{Exosomal SOX2-OT increases proliferation and attenuates apoptosis of ovarian cancer cells}

Next, the role of exosomal SOX2-OT in ovarian cancer was analyzed. The exosome was extracted from the culture medium of TOV-21G and SKOV-3 cells and the characteristic were shown by TEM (Figure 2A). Besides, the expression levels of CD63 and CD9 were identified in the exosome of the TOV$21 \mathrm{G}$ and SKOV-3 cells (Figure 2B). In addition, the SOX2-OT expression was tested in culture medium treated with RNase A or co-treated with RNase A and Triton X-100. Our data showed that the SOX2-OT expression was unacted on the treatment of RNase A while significantly reduced upon the simultaneous cotreatment of RNase A and Triton X-100 (Figure 2C). Next, the efficiency of SOX2-OT depletion was validated in the TOV-21G and SKOV-3 cells (Figure 2D). Significantly, the cell proliferation was reduced by the depletion of SOX2-OT in the cells (Figure 2E). Meanwhile, the colony formation assays confirmed the similar results in the cells (Figure 2F). Moreover, the SOX2-OT knockdown induced the apoptosis of TOV-21G and SKOV-3 cells (Figure $2 \mathrm{G}, 2 \mathrm{H})$. Consistently, the depletion of SOX2-OT reduced Bcl-2 expression while enhanced Bax and cleaved-caspase 3 expression in the cells (Figure 2I).

\section{Exosomal SOX2-OT induces invasion and migration}

Then, we revealed that TOV-21G and SKOV-3 cell invasion and migration were increased by SOX2-OT knockdown (Figure 3A, 3B). Similarly, the depletion of SOX2-OT significantly enhanced wound healing proportion (Figure 3C, 3D), indicating that exosomal SOX2-OT induces invasion and migration.

\section{SOX2-OT serves as a miR-181b-5p sponge in ovarian cancer cells}

The binding region between SOX2-OT and miR$181 b-5 p$ was predicted in the bioinformatic analysis (Figure 4A). The effectiveness of miR-181b-5p mimic was confirmed in TOV-21G and SKOV-3 cells (Figure 4B). The luciferase activities of SOX2-OT, but not SOX2-OT mutant, were decreased by miR- 
181b-5p mimic in the cells (Figure 4C, 4D). Consistently, SOX2-OT knockdown down-regulated the miR-181b-5p expression in the TOV-21G and SKOV-3 cells, suggesting that SOX2-OT serves as a miR-181b-5p sponge in ovarian cancer cells (Figure 4E).

\section{MiR-181b-5p targets SCD1 in ovarian cancer cells}

The miR-181b-5p-targeted site in SCD1 3' UTR was found in a bioinformatic analysis (Figure 5A). Significantly, the luciferase activities of SCD1, but not SCD1 mutant, were reduced by miR-181b-5p mimic in the TOV-21G and SKOV-3 cells (Figure $5 \mathrm{~B})$. Moreover, the expression level of SCD1 was remarkably down-regulated by $\mathrm{miR}-181 \mathrm{~b}-5 \mathrm{p}$ mimic in the cells (Figure 5C, 5D). In addition, the levels of SCD1 was up-regulated by SOX2-OT knockdown, while the miR-181b-5p inhibitor could reverse this effect in the TOV-21G and SKOV-3 cells (Figure $5 \mathrm{E}, 5 \mathrm{~F})$.

\section{Exosomal SOX2-OT contributes to the progression of ovarian cancer by miR-181b-5p/SCD1 axis in vitro}

Next, we investigated the effect of the exosomal SOX2-OT/miR-181b-5p/SCD1 axis in ovarian cancer progression. The exosomal depletion of SOX2-OT reduced the viability of TOV-21G and SKOV-3 cells, and the miR-181b-5p inhibitor or SCD1 overexpression rescued the phenotype (Figure 6A, 6B). Moreover, the miR-181b-5p inhibitor or SCD1 overexpression was able to reverse the SOX2-OTdepletion-induced apoptosis of TOV-21G and SKOV3 cells (Figure 6C, 6D). Consistently, the depletion of SOX2-OT reduced Bcl-2 expression while enhanced
Bax and cleaved-caspase 3 expression in the cells, in which miR-181b-5p inhibitor or SCD1 reversed the effect (Figure 6E).

\section{Exosomal SOX2-OT promotes tumor growth of ovarian cancer in vivo}

We then evaluated the role of exosomal SOX2-OT in the ovarian cancer progression in vivo by the tumorigenicity analysis. The expression of inhibited SOX2-OT was validated in the system (Figure 7A). Significantly, the exosomal SOX2-OT depletion could attenuate the tumor size, volume, and weight (Figure 7B-7D). MiR-181b-5p expression was increased but the SCD1 expression down-regulated in tumor tissues (Figure 7E, 7F), indicating that exosomal SOX2-OT promotes tumor growth of ovarian cancer in vivo.

\section{DISCUSSION}

Ovarian cancer is the predominant type of gynecologic cancer with severe morbidity and high mortality [1]. Exosomes-derived lncRNAs play critical roles in ovarian cancer [13]. Nevertheless, the function of exosomal SOX2-OT in ovarian cancer development. In the present investigation, we found that exosomal SOX2-OT promoted ovarian cancer malignant phenotype by modulating miR-181b$5 \mathrm{p} / \mathrm{SCD} 1$ axis.

It has been found that several exosomal non-coding RNAs are involved in the regulation of ovarian cancer. Exosome-derived miR-205 enhances metastasis of ovarian cancer cells by promoting angiogenesis [29]. Circular RNA WHSC1 contributes to progression of ovarian cancer by modulating hTERT
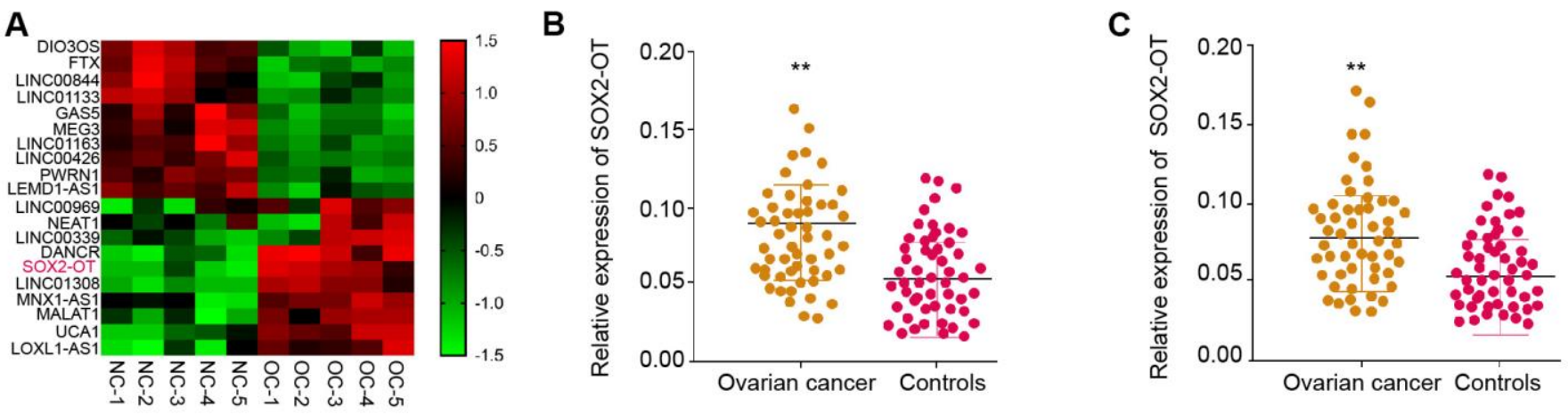

Figure 1. The expression of SOX2-OT is enhanced in the plasma exosome of ovarian cancer patients. (A) The IncRNA profiling was performed in the exosomes from ovarian cancer patients $(n=5)$ and normal controls $(n=5)$. (B) The expression of SOX2-OT was tested by qPCR in the plasma exosome from ovarian cancer patients $(n=55)$. (C) The expression of SOX2-OT was analyzed by qPCR in the ovarian cancer patients $(n=55)$ and the adjacent normal tissues $(n=55)$. 

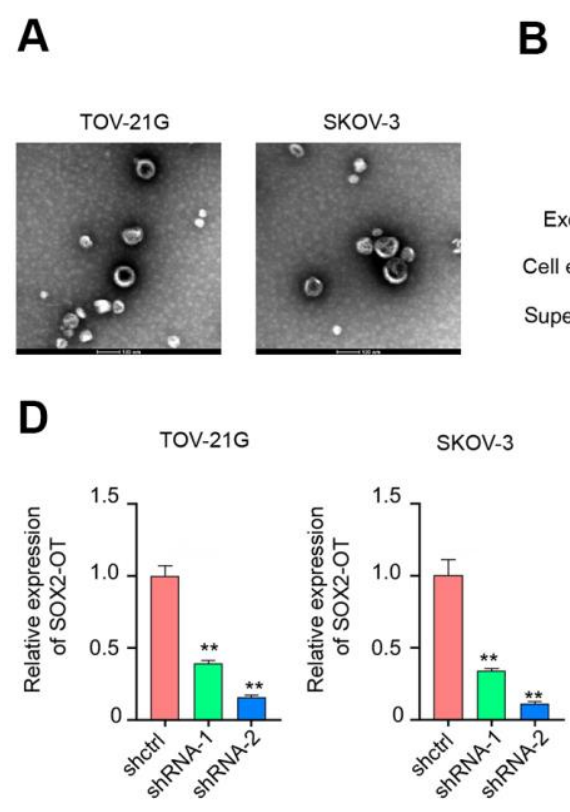

G
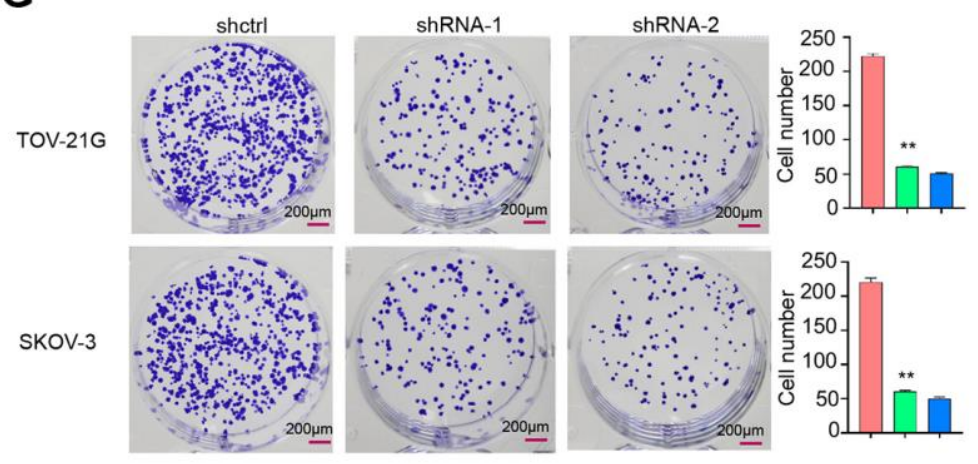

H

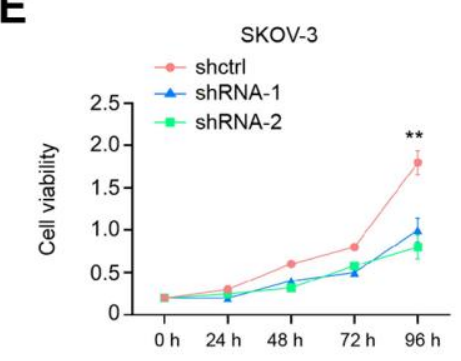

$\mathbf{F}$
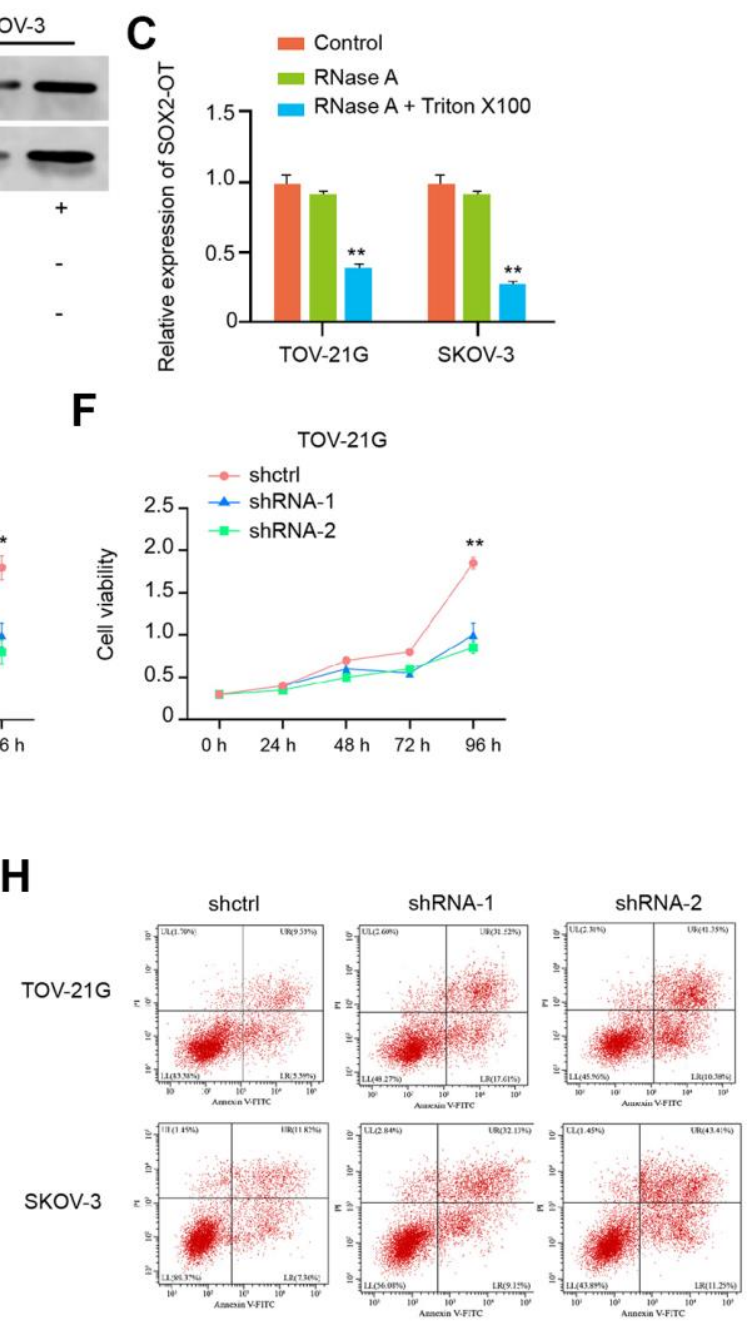

\section{I}
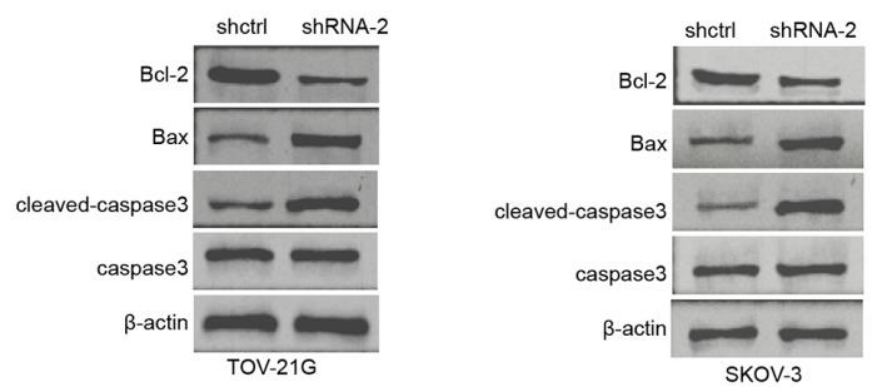

Figure 2. Exosomal SOX2-OT increases proliferation and attenuates apoptosis of ovarian cancer cells. (A) The characteristics of exosomes were measured by the TEM in the TOV-21G and SKOV-3 cells. (B) The expression of CD9 and CD63 was tested by the Western blot analysis in the exosome of TOV-21G and SKOV-3 cells. (C) The expression of SOX2-OT was analyzed by qPCR in the TOV-21G and SKOV-3 cells treated with RNase A $(1 \mu \mathrm{g} / \mathrm{mL})$ or co-treated with RNase A $(1 \mu \mathrm{g} / \mathrm{mL}) \mathrm{and}$ Triton X100 $(0.1 \%)$. (D-I) The TOV-21G and SKOV-3 cells were treated with the SOX2-OT shRNA or control shRNA, and the exosomes were extracted and further treated the cells. (D) The efficiency of the SOX2-OT knockdown was confirmed by qPCR assays in the cells. (E, F) The cell viability was assessed by the MTT assays in the cells. (G) The cell proliferation was determined by colony formation assays in the cells. (H) The cell apoptosis was examined by flow cytometry analysis in the cells. (I) The expression of Bcl-2, Bax, cleaved-caspase3, and caspase 3 was measured by Western blot analysis in the cell. Data are presented as mean \pm SD. Statistic significant difference $s$ were indicated: $* P<0.05, * * P<0.01, * * * P<0.001$. 
and MUC1 through targeting exosomal miR-1182 and miR-145 [30]. Exosomal miR21 promotes paclitaxel resistance by targeting APAF1 in ovarian cancer [31]. The epithelial ovarian cancer exosomal IncRNAs suppress migration of ovarian cancer [32]. In this study, we showed that SOX2-OT was up-regulated in the plasma exosome of ovarian cancer patients. Exosomal SOX2-OT enhanced migration, invasion, and proliferation, and repressed apoptosis of ovarian cancer cells. These data indicate an unreported role of exosomal SOX2-OT in ovarian cancer, indicating informative evidence of crucial roles of exosomal lncRNAs in ovarian cancer.

Moreover, IncRNA normally exert their activity by interacting with miRNAs and various miRNAs contribute to the modulation of ovarian cancer. It has been reported that miR-205 enhances invasion of ovarian cancer cells by inhibiting TCF21 [33]. MiR34 represses cell proliferation by facilitating apoptosis and autophagy and suppresses cell invasion by regulating Notch1 in ovarian cancer [34]. MiR-204-5p attenuates cell growth by decreasing USP47 expression in ovarian cancer [35]. Furthermore, it has been identified that SCD1 is able to regulate tumorigenesis. SCD1 elevates migration of the triplenegative breast cancer cells by regulating $\mathrm{PLD} / \mathrm{mTOR}$ axis [36]. SCD1 enhances metastasis by inhibiting PTEN in glucose response of colorectal cancer [37]. SCD1 controls the stemness process by targeting YAP/TAZ signaling in lung cancer [38]. It also has been found that SCD1 preserves ovarian cancer cells against ferroptosis [28]. Our mechanism research revealed that SOX2-OT sponged miR-181b-5p and
A

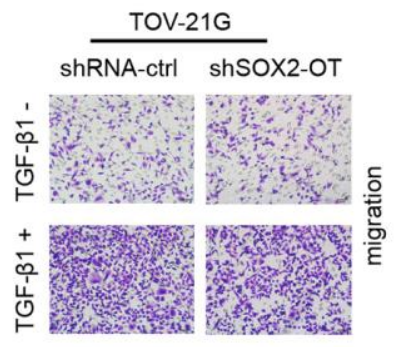

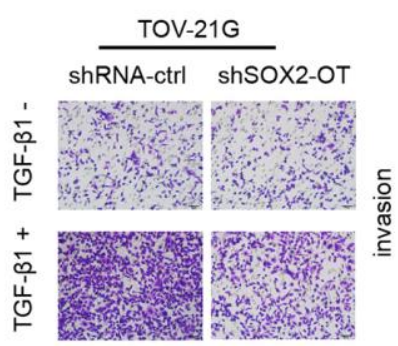

B

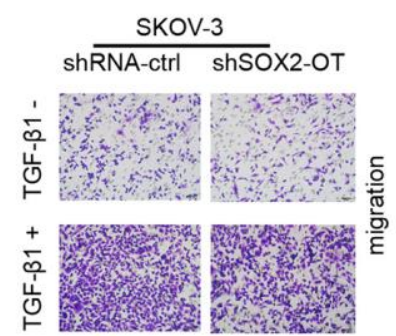

\section{C}
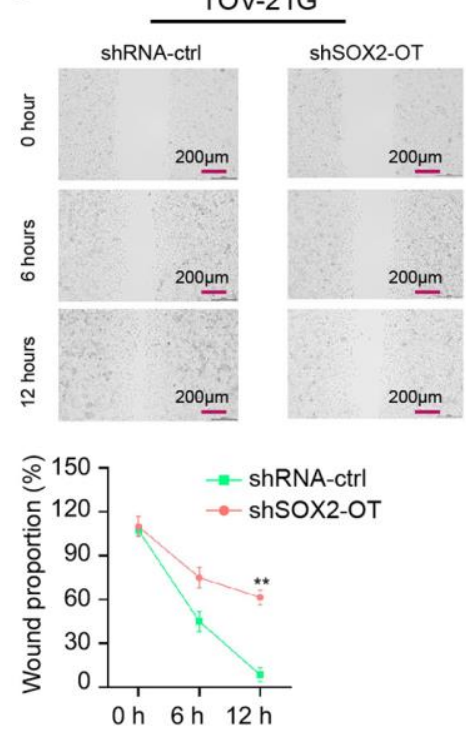

D
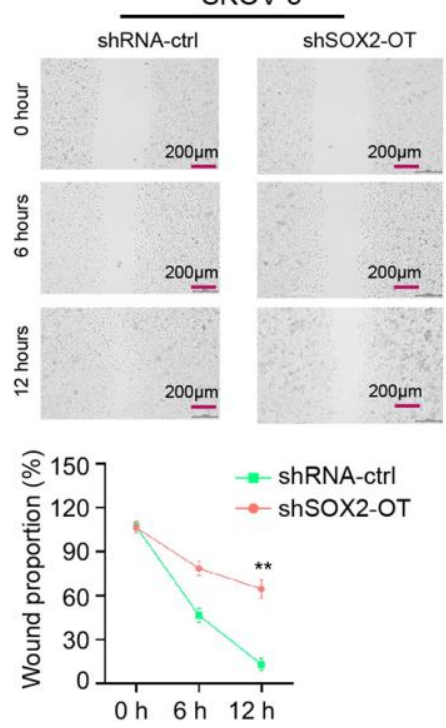

Figure 3. Exosomal SOX2-OT induces invasion and migration of ovarian cancer cells. (A-D) The TOV-21G and SKOV-3 cells were treated with control shRNA or SOX2-OT shRNA, and the exosomes were extracted and further treated the cells. (A, B) The cells were treated with or without TGF- $\beta 1(5 \mathrm{ng} / \mathrm{ml})$. The cell migration and invasion were analyzed by transwell assays in the cells. (C, D) The migration and invasion were determined by wound healing assays in the cells. The wound healing proportion was shown. Data are presented as mean \pm SD. Statistic significant differences were indicated: $* P<0.05, * * P<0.01$. 
A
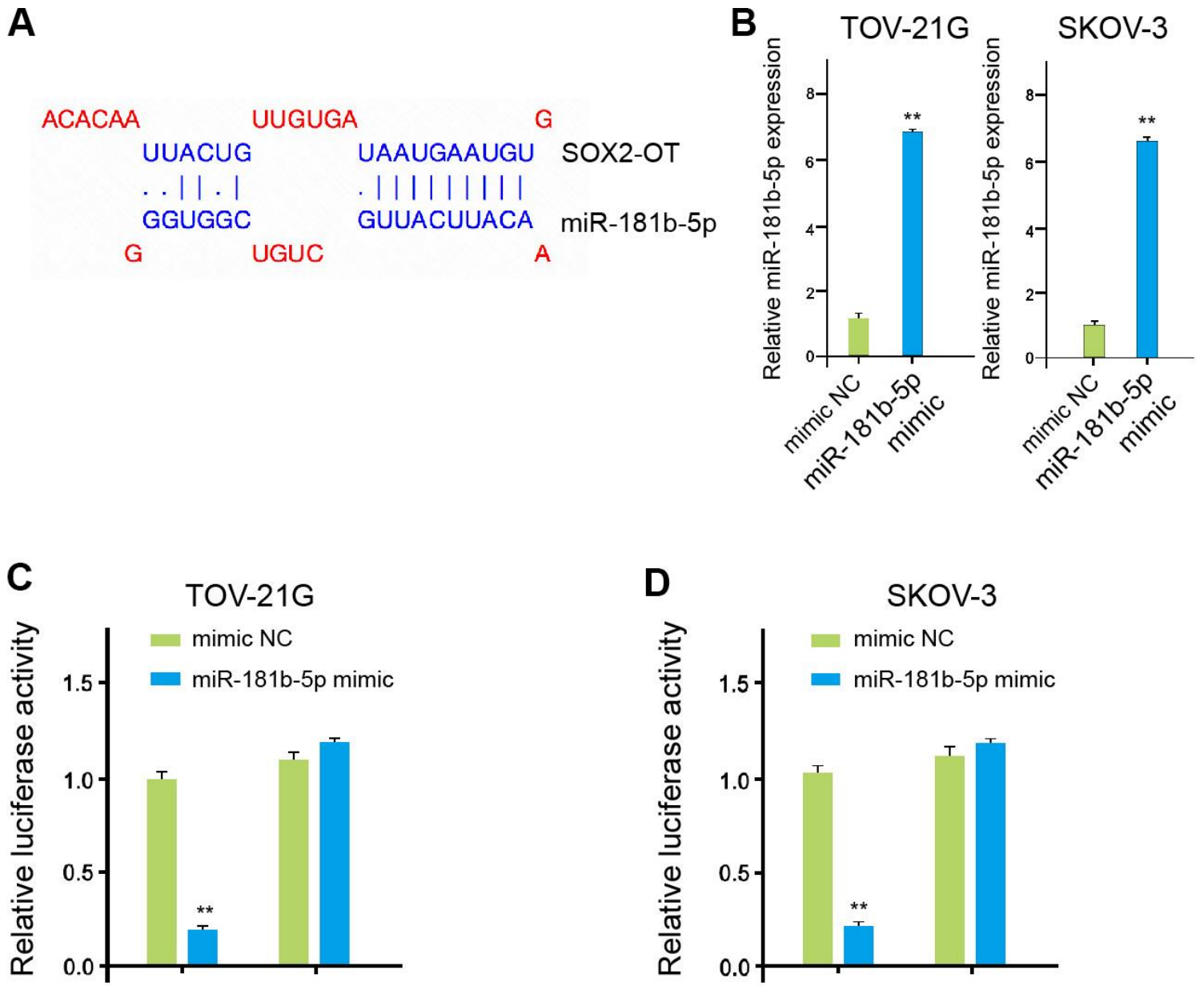

$\mathbf{E}$
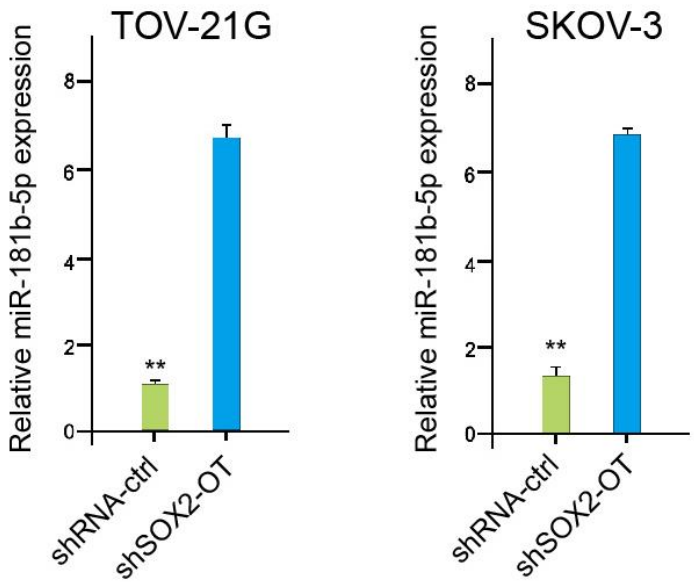

Figure 4. SOX2-OT serves as a miR-181b-5p sponge in ovarian cancer cells. (A) The interaction of SOX2-OT and miR-181b-5p was analyzed by bioinformatic analysis based on ENCORI (http://starbase.sysu.edu.cn/index.php). (B) The expression levels of miR-181b-5p were assessed by qPCR in the TOV-21G and SKOV-3 cells treated with control mimic or miR-181b-5p mimics. (C, D) Luciferase activities of SOX2-OT (SOX2-OT WT) and SOX2-OT with the miR-181b-5p-binding site mutant (SOX2-OT MUT) were determined by luciferase reporter gene assays in the TOV-21G and SKOV-3 cells treated with control mimic or miR-181b-5p mimic. (E) The TOV-21G and SKOV-3 cells were treated with control shRNA or SOX2-OT shRNA. The expression of miR-181b-5p was measured by qPCR assays in the cells. Data are presented as mean \pm SD. Statistic significant differences were indicated: $* * P<0.01$ 


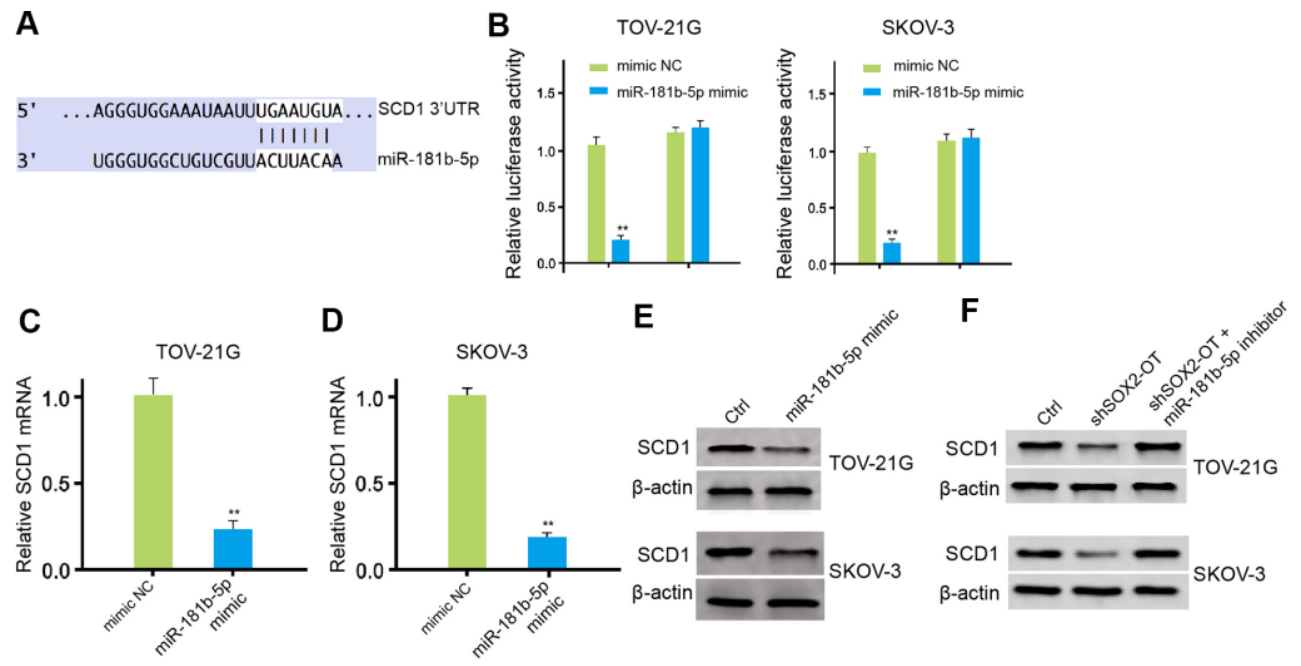

Figure 5. MiR-181b-5p targets SCD1 in ovarian cancer cells. (A) The binding of miR-181b-5p and SCD1 3' UTR was analyzed by bioinformatic analysis based on Targetscan (http://www.targetscan.org/vert 72/). (B-D) The TOV-21G and SKOV-3 cells were treated with control mimic or miR-181b-5p mimic. (B) The luciferase activities of wild type SCD1 (SCD1 WT) and SCD1 with the miR-181b-5p-binding site mutant (SCD1 MUT) were determined by luciferase reporter gene assays in the cells. (C, D) The mRNA expression of SCD1 was tested by qPCR assays in the cells. (E, F) The protein expression of SCD1 was measured by Western blot analysis in the cells. The TOV-21G and SKOV-3 cells were treated with control shRNA or SOX2-OT shRNA, co-treated with SOX2-OT shRNA and miR-181b-5p inhibitor. The protein expression of SCD1 was analyzed by Western blot analysis in the cells. Data are presented as mean \pm SD. Statistic significant differences were indicated: ${ }^{*} P<0.05, * * P<0.01$.

A

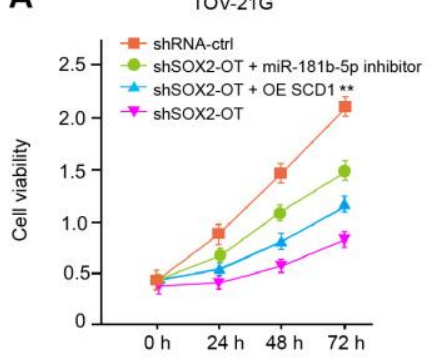

C

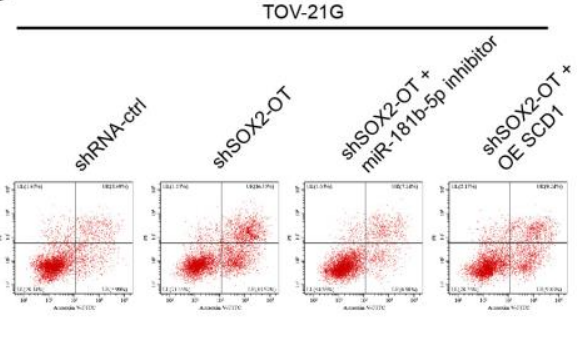

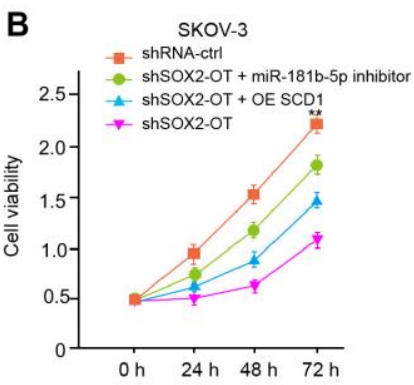

D

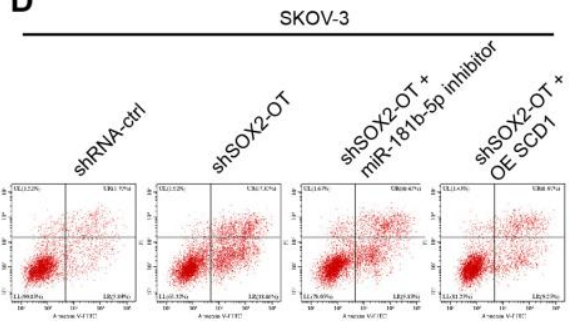

E
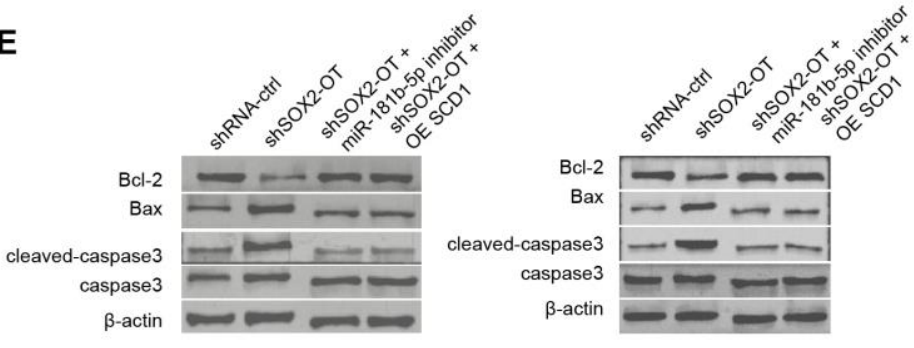

Figure 6. Exosomal SOX2-OT contributes to the progression of ovarian cancer by miR-181b-5p/ SCD1 axis in vitro. (A-E) The TOV-21G and SKOV-3 cells were treated with control shRNA or SOX2-OT shRNA, co-treated with SOX2-OT shRNA and miR-181b-5p inhibitor 
or pcDNA3.1-SCD1. (A, B) The cell viability was measured by MTT assays in the cells. (C, D) The cell apoptosis was analyzed by flow cytometry analysis in the cells. (E) The expression of Bcl-2, Bax, cleaved-caspase3, and caspase3 was measured by Western blot analysis in the cell. Data are presented as mean \pm SD. Statistic significant differences were indicated: $* * P<0.01$.

miR-181b-5p targeted SCD1 in ovarian cancer cells. The SCD1 overexpression and miR-181b-5p inhibitor could reverse exosomal SOX2-OT-mediated ovarian cancer progression. It uncovers a novel function of SOX2-OT/miR-181b-5p/SCD1 axis in ovarian cancer development, presenting the unreported mechanism involving SOX2-OT, miR-181b-5p, and SCD1.

In conclusion, we identified that exosomal SOX2-OT contributed to ovarian cancer malignant phenotype by miR-181b-5p/SCD1 axis (Figure 7G). Our finding
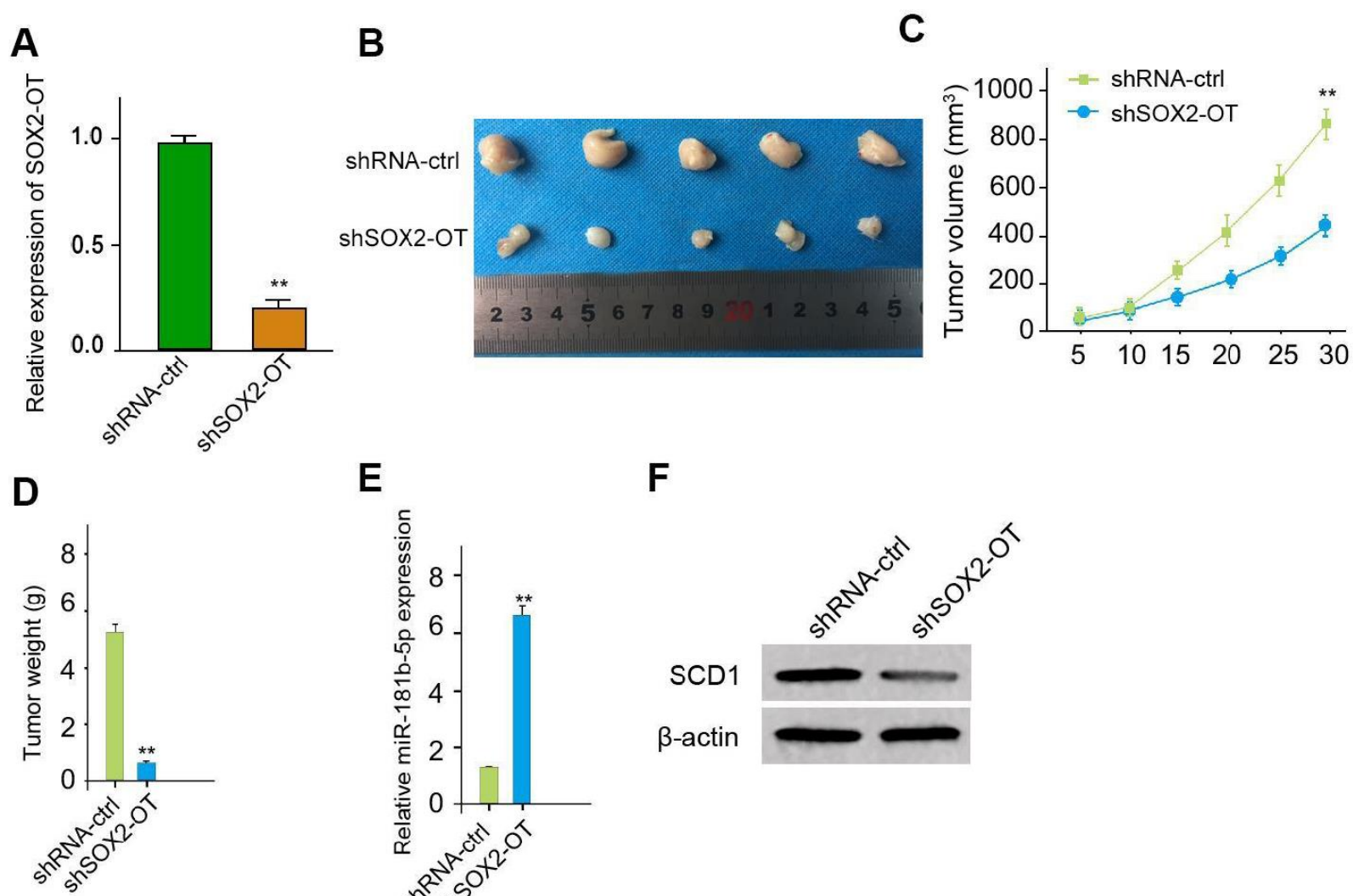

E

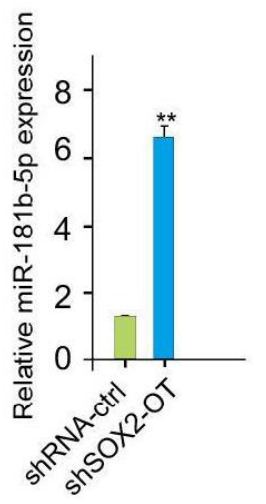

$\mathbf{F}$

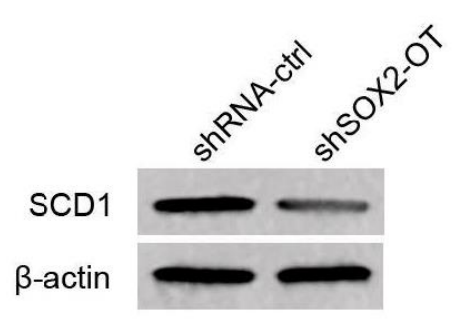

G

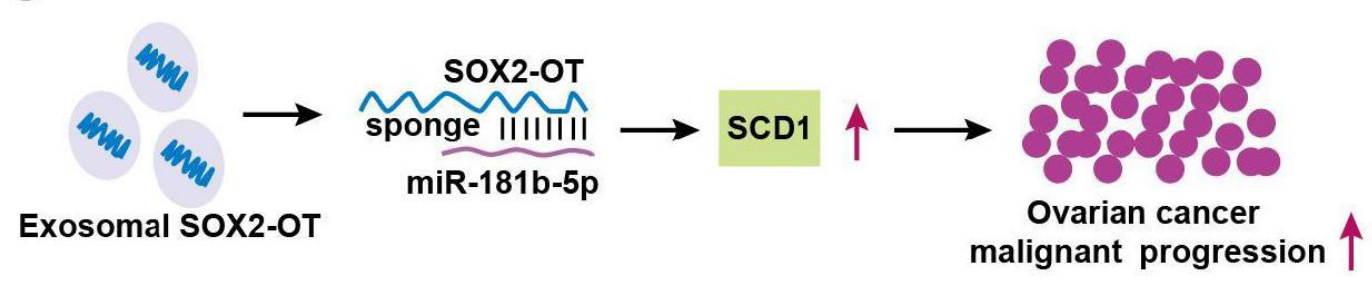

Figure 7. Exosomal SOX2-OT promotes tumor growth of ovarian cancer in vivo. (A-F) The impact of exosomal SOX2-OT on tumor growth of ovarian cancer cells in vivo was analyzed by nude mice tumorigenicity assay $(n=5)$. The SKOV-3 cells were treated with control shRNA or SOX2-OT shRNA exosome. (A) The expression of SOX2-OT was tested by qPCR in the tumor tissues of the mice. (B) Representative images of dissected tumors from nude mice were shown. (C) The average tumor volume was calculated and presented. (D) The average tumor weight was calculated and presented. (E) The expression of miR-181b-5p was measured by qPCR in the tumor tissues of the mice. (F) The expression of SCD1 was tested by Western blot analysis in the tumor tissues of the mice. (G) The schematic model of the study. Data are presented as mean \pm SD. Statistic significant differences were indicated: $* P<0.05, * *$ $P<0.01$. 
describes novel insights in the mechanism that exosomal lncRNA SOX2-OT promotes ovarian cancer progression. SOX2-OT, miR-181b-5p and SCD1 may be utilized as therapeutic targets.

\section{MATERIALS AND METHODS}

\section{Ovarian cancer clinical samples}

The 55 ovarian cancer clinical samples were collected from People's Hospital of Rizhao. All the cases were detected by histopathological measurement and performed the diagnosis by two clinicians. Samples collected from the patients and stored at $-80^{\circ} \mathrm{C}$. The IncRNA profiling was performed (Annoroad, China). The samples applied in this research were under the written approval of the cases. All experiments are consistent with guidelines of Ethics Committee of People's Hospital of Rizhao.

\section{Exosome isolation and analysis}

The Exosomes were isolated and verified as the previous report [39]. The plasma and culture medium were centrifuged for 15 minutes at the condition of $3000 \times \mathrm{g}$ to eliminate cell debris. Then, the exosomes were extracted by an Exoquick exosome precipitation solution (System Biosciences, USA). The exosomes were observed and validated using the transmission electron microscopy (TEM, Hitachi, Japan).

\section{Cell culture and treatment}

The TOV-21G and SKOV-3 cells were maintained in the lab. The TOV-21G and SKOV-3 cells were incubated at the incubator of $5 \% \mathrm{CO}_{2}$ and $37^{\circ} \mathrm{C}$ in the DMEM medium (Hyclone, USA) with penicillin (100 units $/ \mathrm{mL}$, Hyclone, USA), streptomycin $(0.1 \mathrm{mg} / \mathrm{mL}$, Hyclone, USA), and FBS (10\%, Hyclone, USA). The control shRNA, SOX2-OT shRNA, pcDNA3.1-SCD1, mimic and inhibitor of miR-181b-5p were purchased from GenePharma (China). SOX2-OT shRNA-1: GCACCGCTATACAGAGAAACCTTATCCTCGAGG ATAAGGTTTCTCTGTATAGCTTTTTTG; SOX2-OT shRNA-2: GCACCGGAGCAAAGGTGCTGTCATTT CTCGAGAAATGACAGCACCTTTGCTCCTTTTTG.

\section{Quantitative reverse transcription-PCR (qRT-PCR)}

Cells were collected after indicated treatment, and total RNA was extracted using TRIZOL (Invitrogen, USA), and reversely transcribed to cDNA using a Reverse Transcription System Ki (TaKaRa, China). The expression was determined by a SYBR Mix kit (Takara, China) in a Real-time PCR system (BD Biosciences, USA) and analyzed using $2^{-\Delta \Delta \mathrm{Ct}}$ method. Primer sequences: SOX2-OT F: 5'-GTTCATGGCCTGGA CTCTCC-3', R: 5'-ATTGCTAGCCCTCACACCTC-3'; miR-181b-5p F: 5'-CTCAACTGGTGTCGTGGAG TCGG-3', R: 5'-CAATTCAGTTGAGTTGCATTC-3'; SCD1 F: 5'- AAACCTGGCTTGCTGATG-3', R: 5'GGGGGCTAATGTTCTTGTCA-3'; GAPDH F: 5'AAGAAGGTGGTGAAGCAGGC-3'， R: 5'-TCCAC CACCCAGTTGCTGTA-3'; U6 F: 5'-GCTTCGGCA GCACATATACTAA-3', R: 5'-AACGCTTCACGAAT TTGCGT-3'.

\section{MTT assay}

The cell viability of TOV-21G and SKOV-3 cells was assessed by a 3-(4, 5- dimethylthiazol-2-yl)-2,5diphenyltetrazolium bromide (MTT) experiment. TOV$21 \mathrm{G}$ and SKOV-3 cells were treated as indicated in each experiment, digested, suspended as single cells, and planted in $96-$ well plates $(3 \times 103$ cells per well). MTT reagent were added at a final concentration of $5 \mathrm{mg} / \mathrm{ml}$ in each well. Following a 4-hours incubation, the cell medium was discarded and replaced by $100 \mu \mathrm{DMSO}$ in each well. The plates were gently shaken in dark for 10 minutes. Absorbance at $490 \mathrm{~nm}$ was detected.

\section{Colony formation assays}

TOV-21G and SKOV-3 cells were planted into 6-well plates with 1000 cells in each well after appropriate transfection, and cultured in $37^{\circ} \mathrm{C}$ incubator for two weeks. After the visible colonies formed, cells were stained with crystal violet diluted in methanol for 20 minutes. The colonies were captured and counted via a microscope (Olympus, Japan).

\section{Transwell assays}

The migration and invasion ability of TOV-21G and SKOV-3 cells were determined via using a transwell chamber (Corning, USA). The cells were treated with or without TGF- $\beta 1(5 \mathrm{ng} / \mathrm{ml})$. To detect migration, TOV$21 \mathrm{G}$ and SKOV-3 $(2 \times 104$ cells/well $)$ were seeded into the upper chambers with FBS-free medium, while the lower chambers were filled with complete DMEM medium. After 24 hours incubation, the membranes of upper chambers were fixed by $4 \%$ paraformaldehyde for $15 \mathrm{~min}$ and stained by $0.5 \%$ crystal violet for 30 minutes. The migrated cells were photographed and counted. For cell invasion, the process was similar with that of migration experiment, only that the upper chambers were coated with Matrigel (BD Bioscience, USA).

\section{Wound healing assay}

TOV-21G and SKOV-3 cells were planted in 4-well plate at a density of $3 \times 105 /$ well to form a monoplayer. 
A sterilized $200 \mathrm{ul}$ pipette were used to gently scratch a line on the monoplayer. Then the cells were washed with PBS to wash out the detached cells, and replaced with fresh FBS-free medium for continuing incubation. The images of scratch were captured at $0 \mathrm{~h}, 6 \mathrm{~h}$ and $12 \mathrm{~h}$ after scratching with a microscope (Olympus), and measured.

\section{Analysis of cell apoptosis}

The transfected TOV-21G and SKOV-3 cells were digested and washed, suspended in $100 \mu \mathrm{L}$ binding buffer and stained with Annexin V-FITC and PI staining reagents under the instruction of detection kit (Keygen, China) $\mathrm{n}$ dark condition for 20 minutes. Afterward, the cells were washed and resuspended in binding buffer and measured by a flow cytometry (BD Biosciences, USA) immediately.

\section{Luciferase reporter gene assay}

The pmirGLO-SCD1, pmirGLO-SCD1 mutant or pmirGLO-SOX2-OT, pmirGLO-SOX2-OT mutant, and miR-181b-5p mimic or control mimic were synthesized and obtained (GenePharma, China). Briefly, the cells were transfected with the pmirGLO-SCD1, pmirGLOSCD1 mutant or pmirGLO-SOX2-OT, pmirGLOSOX2-OT mutant, and miR-181b-5p mimic or control mimic. Renilla was applied as a normalized control.

\section{Western blot analysis}

FTC-133 and TPC-1 cells received indicated treatment were washed in PBS and lysed with ice-cold lysis buffer containing a cocktail of proteinase inhibitors. Total protein was divided in SDS-PAGE gel and shifted to $\mathrm{NC}$ membranes. The membranes were soaked in fast blocking buffer for 15 minutes and incubated with specific primary antibodies, namely the TSG101 (Abcam, USA), CD63 (Abcam, USA), Grp94 (Abcam, USA), CD9 (Abcam, USA), SCD1 (Abcam, USA), Bcl2 (Abcam, USA), Bax (Abcam, USA), cleavedcaspase3 (Abcam, USA), caspase3 (Abcam, USA), and $\beta$-actin (Abcam, USA), at $4^{\circ} \mathrm{C}$ overnight. Next day, the membranes were incubated with corresponding HRPconjugated secondary antibodies and ECL substrate (Beyotime, China). The visualization of proteins was performed by a Gel imaging system (BD Biosciences, USA).

\section{Analysis of tumorigenicity in nude mice}

All animal experiments in this work were authorized by Ethics Committee of People's Hospital of Rizhao. $\mathrm{BALB} / \mathrm{c}$ nude mice aged 4-week were maintained in a specific pathogen-free (SPF) environment. The mice were randomly divided into two groups $(n=5)$. A total number of $1 \times 107$ SKOV-3 cells transfected with control shRNA or SOX2-OT shRNA exosome were collected, washed, suspended in $100 \mu \mathrm{L}$ saline, and then subcutaneously injected into the left fat pad of each mice. The tumor size (volume $=0.5 \times$ width $2 \times$ length) and body weight were measured at the indicated time points.

\section{Statistical analysis}

Data in this study were repeated at least three times, shown as means \pm SD and analyzed by a GraphPad prism 7 software. The statistical significance were defined by $\mathrm{p}<0.05$ in Student's t test or one-way ANNOVA analysis.

\section{AUTHOR CONTRIBUTIONS}

Yongjing Lai and Lihua Dong performed the majority of experiments, collected and analyzed the data; Huifang Jin performed the molecular investigations; Hongju $\mathrm{Li}$ designed and coordinated the research; Meiling Sun and Jianlan Li wrote the paper.

\section{CONFLICTS OF INTEREST}

The authors declare that they have no conflicts of interest.

\section{FUNDING}

This research received support from People's Hospital of Rizhao.

\section{REFERENCES}

1. Webb PM, Jordan SJ. Epidemiology of epithelial ovarian cancer. Best Pract Res Clin Obstet Gynaecol. 2017; 41:3-14. https://doi.org/10.1016/j.bpobgyn.2016.08.006 PMID:27743768

2. Gao MQ, Choi YP, Kang S, Youn JH, Cho NH. CD24+ cells from hierarchically organized ovarian cancer are enriched in cancer stem cells. Oncogene. 2010; 29:2672-80. https://doi.org/10.1038/onc.2010.35 PMID:20190812

3. Lalremmawia H, Tiwary BK. Identification of molecular biomarkers for ovarian cancer using computational approaches. Carcinogenesis. 2019; 40:742-48. https://doi.org/10.1093/carcin/bgz025 PMID:30753333

4. van Dam GM, Themelis G, Crane LM, Harlaar NJ, Pleijhuis RG, Kelder W, Sarantopoulos A, de Jong JS, Arts HJ, van der Zee AG, Bart J, Low PS, Ntziachristos V. 
Intraoperative tumor-specific fluorescence imaging in ovarian cancer by folate receptor- $\alpha$ targeting: first inhuman results. Nat Med. 2011; 17:1315-19. https://doi.org/10.1038/nm.2472 PMID:21926976

5. Delort L, Kwiatkowski F, Chalabi N, Satih S, Bignon YJ, Bernard-Gallon DJ. Central adiposity as a major risk factor of ovarian cancer. Anticancer Res. 2009; 29:5229-34.

PMID:20044641

6. Bull CJ, Yarmolinsky J, Wade KH. Commentary: Mendelian randomization analysis identifies circulating vitamin $\mathrm{D}$ as a causal risk factor for ovarian cancer. Int J Epidemiol. 2016; 45:1631-33. https://doi.org/10.1093/ije/dyw265 PMID:27892412

7. Kazerouni N, Greene MH, Lacey JV Jr, Mink PJ, Schairer C. Family history of breast cancer as a risk factor for ovarian cancer in a prospective study. Cancer. 2006; 107:1075-83.

https://doi.org/10.1002/cncr.22082 PMID:16881078

8. Tomasetti M, Lee W, Santarelli L, Neuzil J. Exosomederived microRNAs in cancer metabolism: possible implications in cancer diagnostics and therapy. Exp Mol Med. 2017; 49:e285.

https://doi.org/10.1038/emm.2016.153 PMID:28104913

9. Fan $Q$, Yang $L$, Zhang $X$, Peng $X$, Wei S, Su D, Zhai Z, Hua $X$, Li $\mathrm{H}$. The emerging role of exosome-derived noncoding RNAs in cancer biology. Cancer Lett. 2018; 414:107-15.

https://doi.org/10.1016/i.canlet.2017.10.040

PMID:29107112

10. Milane L, Singh A, Mattheolabakis G, Suresh M, Amiji MM. Exosome mediated communication within the tumor microenvironment. J Control Release. 2015; 219:278-94.

https://doi.org/10.1016/i.jconrel.2015.06.029 PMID:26143224

11. He C, Zheng S, Luo Y, Wang B. Exosome Theranostics: Biology and Translational Medicine. Theranostics. 2018; 8:237-55.

https://doi.org/10.7150/thno.21945 PMID:29290805

12. Shen J, Zhu X, Fei J, Shi P, Yu S, Zhou J. Advances of exosome in the development of ovarian cancer and its diagnostic and therapeutic prospect. Onco Targets Ther. 2018; 11:2831-41. https://doi.org/10.2147/OTT.S159829 PMID:29844681

13. Nakamura K, Sawada K, Kobayashi M, Miyamoto M, Shimizu A, Yamamoto $M$, Kinose $Y$, Kimura T. Role of the Exosome in Ovarian Cancer Progression and Its Potential as a Therapeutic Target. Cancers (Basel). 2019; 11:1147. https://doi.org/10.3390/cancers11081147 PMID:31405096

14. Wo Q, Zhang D, Hu L, Lyu J, Xiang F, Zheng W, Shou J, Qi X. Long noncoding RNA SOX2-OT facilitates prostate cancer cell proliferation and migration via miR-3693p/CFL2 axis. Biochem Biophys Res Commun. 2019; 520:586-93.

https://doi.org/10.1016/i.bbrc.2019.09.108 PMID: $\underline{1623830}$

15. Zhang E, Li X. LncRNA SOX2-OT regulates proliferation and metastasis of nasopharyngeal carcinoma cells through miR-146b-5p/HNRNPA2B1 pathway. J Cell Biochem. 2019; 120:16575-88. https://doi.org/10.1002/jcb.28917 PMID:31099048

16. Teng $\mathrm{Y}$, Kang $\mathrm{H}$, Chu $\mathrm{Y}$. Identification of an Exosomal Long Noncoding RNA SOX2-OT in Plasma as a Promising Biomarker for Lung Squamous Cell Carcinoma. Genet Test Mol Biomarkers. 2019; 23:23540.

https://doi.org/10.1089/gtmb.2018.0103 PMID: $\underline{0986097}$

17. Ling $\mathrm{H}$, Wei $\mathrm{Z}$. The clinical significance of long non coding RNA SOX2-OT in ovarian cancer tissues and cell lines. Chinese Journal of cancer prevention and treatment. 2019; 26:100-06.

18. Lu TX, Rothenberg ME. MicroRNA. J Allergy Clin Immunol. 2018; 141:1202-07.

https://doi.org/10.1016/j.jaci.2017.08.034 PMID:29074454

19. Rupaimoole R, Slack FJ. MicroRNA therapeutics: towards a new era for the management of cancer and other diseases. Nat Rev Drug Discov. 2017; 16:203-22. https://doi.org/10.1038/nrd.2016.246 PMID:28209991

20. Xiang G, Cheng Y. MiR-126-3p inhibits ovarian cancer proliferation and invasion via targeting PLXNB2. Reprod Biol. 2018; 18:218-24.

https://doi.org/10.1016/i.repbio.2018.07.005 PMID:30054097

21. Lv T, Song K, Zhang L, Li W, Chen Y, Diao Y, Yao Q, Liu P. miRNA-34a decreases ovarian cancer cell proliferation and chemoresistance by targeting HDAC1. Biochem Cell Biol. 2018; 96:663-71.

https://doi.org/10.1139/bcb-2018-0031 PMID:29561664

22. Han Z, Zhan R, Chen S, Deng J, Shi J, Wang W. miR$181 \mathrm{~b} /$ Oncostatin $\mathrm{m}$ axis inhibits prostate cancer bone metastasis via modulating osteoclast differentiation. J Cell Biochem. 2020; 121:1664-74. https://doi.org/10.1002/jcb.29401 PMID:31680294

23. Li X, Han J, Zhu H, Peng L, Chen Z. miR-181b-5p mediates TGF- $\beta 1$-induced epithelial-to-mesenchymal 
transition in non-small cell lung cancer stem-like cells derived from lung adenocarcinoma A549 cells. Int J Oncol. 2017; 51:158-68.

https://doi.org/10.3892/ijo.2017.4007 PMID:28534939

24. Chen S, Liu Y, Wang Y, Xue Z. LncRNA CCAT1 Promotes Colorectal Cancer Tumorigenesis Via A miR-181b5p/TUSC3 Axis. Onco Targets Ther. 2019; 12:9215-25. https://doi.org/10.2147/OTT.S216718 PMID: $\underline{31807005}$

25. Bansal S, Berk M, Alkhouri N, Partrick DA, Fung JJ, Feldstein A. Stearoyl-CoA desaturase plays an important role in proliferation and chemoresistance in human hepatocellular carcinoma. J Surg Res. 2014; 186:29-38.

https://doi.org/10.1016/i.jss.2013.07.001

PMID:24135379

26. Hess D, Chisholm JW, Igal RA. Inhibition of stearoyICoA desaturase activity blocks cell cycle progression and induces programmed cell death in lung cancer cells. PLoS One. 2010; 5:e11394.

https://doi.org/10.1371/journal.pone.0011394 PMID:20613975

27. Mauvoisin D, Charfi C, Lounis AM, Rassart E, Mounier C. Decreasing stearoyl-CoA desaturase-1 expression inhibits $\beta$-catenin signaling in breast cancer cells. Cancer Sci. 2013; 104:36-42.

https://doi.org/10.1111/cas.12032

PMID:23013158

28. Tesfay L, Paul BT, Konstorum A, Deng Z, Cox AO, Lee J, Furdui CM, Hegde P, Torti FM, Torti SV. Stearoyl-CoA Desaturase 1 Protects Ovarian Cancer Cells from Ferroptotic Cell Death. Cancer Res. 2019; 79:5355-66. https://doi.org/10.1158/0008-5472.CAN-19-0369 PMID:31270077

29. He L, Zhu W, Chen Q, Yuan Y, Wang Y, Wang J, Wu X. Ovarian cancer cell-secreted exosomal miR-205 promotes metastasis by inducing angiogenesis. Theranostics. 2019; 9:8206-20.

https://doi.org/10.7150/thno.37455

PMID:31754391

30. Zong ZH, Du YP, Guan X, Chen S, Zhao Y. CircWHSC1 promotes ovarian cancer progression by regulating MUC1 and hTERT through sponging miR-145 and miR1182. J Exp Clin Cancer Res. 2019; 38:437.

https://doi.org/10.1186/s13046-019-1437-z PMID:31666098

31. Au Yeung $C L$, Co NN, Tsuruga $T$, Yeung TL, Kwan SY, Leung CS, Li Y, Lu ES, Kwan K, Wong KK, Schmandt R, Lu $\mathrm{KH}$, Mok SC. Exosomal transfer of stroma-derived miR21 confers paclitaxel resistance in ovarian cancer cells through targeting APAF1. Nat Commun. 2016; 7:11150. https://doi.org/10.1038/ncomms11150 PMID:27021436
32. Wu $Q$, Wu X, Ying $X$, Zhu $Q$, Wang $X$, Jiang $L$, Chen $X$, Wu $Y$, Wang $X$. Suppression of endothelial cell migration by tumor associated macrophage-derived exosomes is reversed by epithelial ovarian cancer exosomal IncRNA. Cancer Cell Int. 2017; 17:62.

https://doi.org/10.1186/s12935-017-0430-x PMID:28592924

33. Wei J, Zhang L, Li J, Zhu S, Tai M, Mason CW, Chapman JA, Reynolds EA, Weiner CP, Zhou HH. MicroRNA-205 promotes cell invasion by repressing TCF21 in human ovarian cancer. J Ovarian Res. 2017; 10:33. https://doi.org/10.1186/s13048-017-0328-1 PMID:28476165

34. Jia $Y$, Lin $R$, Jin $H$, Si L, Jian $W$, Yu $Q$, Yang S. MicroRNA34 suppresses proliferation of human ovarian cancer cells by triggering autophagy and apoptosis and inhibits cell invasion by targeting Notch 1 . Biochimie. 2019; 160:193-99.

https://doi.org/10.1016/i.biochi.2019.03.011 PMID:30905732

35. Hu L, Kolibaba H, Zhang S, Cao M, Niu H, Mei H, Hao Y, Xu Y, Yin Q. MicroRNA-204-5p Inhibits Ovarian Cancer Cell Proliferation by Down-Regulating USP47. Cell Transplant. 2019; 28:51S-8S. https://doi.org/10.1177/0963689719877372 PMID:31526052

36. Lingrand $M$, Lalonde $S$, Jutras-Carignan $A$, Bergeron $K F$, Rassart E, Mounier C. SCD1 activity promotes cell migration via a PLD-mTOR pathway in the MDA-MB231 triple-negative breast cancer cell line. Breast Cancer. 2020; 27:594-606.

https://doi.org/10.1007/s12282-020-01053-8 PMID:31993937

37. Ran $\mathrm{H}$, Zhu $\mathrm{Y}$, Deng $\mathrm{R}$, Zhang $\mathrm{Q}$, Liu $X$, Feng $\mathrm{M}$, Zhong J, Lin S, Tong $X$, Su Q. Stearoyl-CoA desaturase-1 promotes colorectal cancer metastasis in response to glucose by suppressing PTEN. J Exp Clin Cancer Res. 2018; 37:54.

https://doi.org/10.1186/s13046-018-0711-9 PMID:29530061

38. Noto A, De Vitis C, Pisanu ME, Roscilli G, Ricci G, Catizone A, Sorrentino G, Chianese G, TaglialatelaScafati O, Trisciuoglio D, Del Bufalo D, Di Martile M, Di Napoli A, et al. Stearoyl-CoA-desaturase 1 regulates lung cancer stemness via stabilization and nuclear localization of YAP/TAZ. Oncogene. 2017; 36:4573-84. https://doi.org/10.1038/onc.2017.75 PMID:28368399

39. Lu Y, Hou K, Li M, Wu X, Yuan S. Exosome-Delivered LncHEIH Promotes Gastric Cancer Progression by Upregulating EZH2 and Stimulating Methylation of the GSDME Promoter. Front Cell Dev Biol. 2020; 8:571297. https://doi.org/10.3389/fcell.2020.571297 PMID:33163491 


\section{SUPPLEMENTARY MATERIALS}

\section{Supplementary Figure}

A

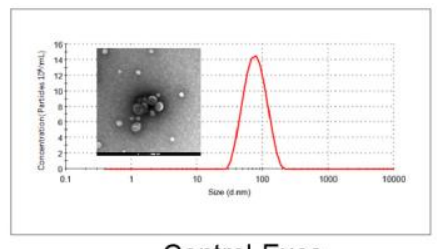

Control-Exos

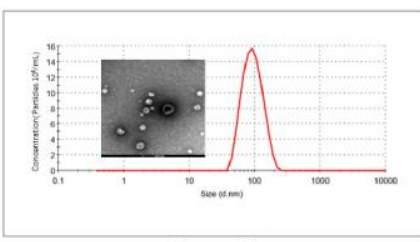

Case-Exos
B

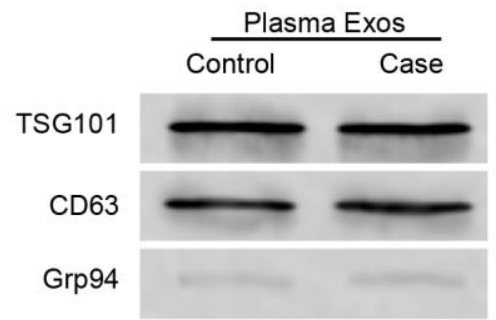

Supplementary Figure 1. The identification of plasma exosome from ovarian cancer patients. (A) The characteristics of exosomes were assessed by the TEM in the ovarian cancer patients. (B) The expression of TSG101, CD63, and Grp94 was measured by Western blot analysis in the exosome from ovarian cancer patients. 